International Journal of Pure and Applied Mathematics

Volume 108 No. 2 2016, 327-339

ISSN: 1311-8080 (printed version); ISSN: 1314-3395 (on-line version)

url: http://www.ijpam.eu

doi: $10.12732 /$ ijpam.v108i2.9

ijpam.eu

\title{
SOME INTEGRAL TRANSFORM OF GENERALIZED MITTAG-LEFFLER FUNCTIONS
}

\author{
Bhausaheb R. Sontakke ${ }^{1}$, Govind P. Kamble ${ }^{2}$, Mohd. Mazhar Ul-Haque ${ }^{3}$ \\ ${ }^{1}$ Department of Mathematics \\ Pratishthan College, Paithan \\ Dist: Aurangabad (M.S.), INDIA \\ ${ }^{2}$ Depatment of Mathematics \\ P.E.S. College of Engineering \\ Nagsenvana, Aurangabad (M.S.), INDIA \\ ${ }^{3}$ Dr. Babasaheb Ambedkar Marathwada University \\ Aurangabad (M.S.), INDIA
}

\begin{abstract}
This paper presents integral transform of Generalized Mittag-Leffler function which plays an important role to solve the differential equations and also some relations and results related to this generalized Mittag-Leffler function. The generalized Mittag-Leffler function arises in the solution of fractional order differential equations and fractional order integral equations.
\end{abstract}

AMS Subject Classification: 26A33, 34K37, 34A08, 45E10, 47H10

Key Words: Laplace transform, Fourier transform, Mittag-Leffler function, generalized Mittag-Leffler function, fractional differential equation, fractional integral equation

\section{Introduction}

Fractional calculus is the branch of mathematics related to the derivatives and integral of integer as well as non-integer order. This branch of mathematics

Received: March 10, 2016

Published: June 20, 2016 (c) 2016 Academic Publications, Ltd.

url: www.acadpubl.eu 
has been applied to almost every field of mathematics, science, engineering and technology, etc. Fractional differential equations have been an active research area during the past few decades and they occur in many applications of Sciences. The Mittag-Leffler function appears as the solution of fractional order differential equations and fractional order integral equations. Some applications of the Mittag-Leffler function are as follows: studies of the kinetic equation, the telegraph equation, random walks, Levy flights, super diffuse transport and complex systems.

Besides this, the Mittag-Leffler function appears in the solution of certain boundary value problems involving fractional integro-differential equations of Voltera type. It has applications in applied problems, such as fluid flow, rheology,diffusive transport, akin to diffusion, electric networks, probability and statistical distribution theory. Various properties of the Mittag-Leffler functions were presented and surveyed. Furthermore, different generalization of the Mittag-Leffler function and its properties have been investigated.

\section{Preliminary Results, Notations and Terminology}

In this section, we present some basic definitions and preliminaries which are useful in further discussion.

Definition 2.1. (Mittag-Leffler Function) [3] The Mittag - Leffler function of one parameter is denoted by $E_{\alpha}(z)$ and defined as,

$$
E_{\alpha}(z)=\sum_{k=0}^{\infty} \frac{1}{\Gamma(\alpha k+1)} z^{k}
$$

where $z, \alpha \in C, \operatorname{Re}(\alpha)>0$.

If we put $\alpha=1$, then the above equation becomes

$$
E_{1}(z)=\sum_{k=0}^{\infty} \frac{z^{k}}{\Gamma(k+1)}=\sum_{k=0}^{\infty} \frac{z^{k}}{k !}=e^{z} .
$$

Definition 2.2. (Mittag-Leffler Function for two parameters) The generalization of $E_{\alpha}(z)$ was studied by Wiman (1905) [13], Agarwal [1] and Humbert and Agarwal [5] defined the function as,

$$
E_{\alpha, \beta}(z)=\sum_{k=0}^{\infty} \frac{1}{\Gamma(\alpha k+\beta)} z^{k}
$$

where $z, \alpha, \beta \in C, \operatorname{Re}(\alpha)>0, \operatorname{Re}(\beta)>0$, 
In 1971, The more generalized function is introduced by Prabhakar [9] as

$$
E_{\alpha, \beta}^{\gamma}\left((z)=\sum_{k=0}^{\infty} \frac{(\gamma)_{k} z^{k}}{\Gamma(\alpha k+\beta)}\right.
$$

where $z, \alpha, \beta, \gamma \in C, \operatorname{Re}(\alpha)>0, \operatorname{Re}(\beta)>0, \operatorname{Re}(\gamma)>0$, where $\gamma \neq 0, \gamma)_{k}=\gamma(\gamma+1)(\gamma+2) \ldots(\gamma+k-1)$ is the Pochhammer symbol [11], and

$$
(\gamma)_{k}=\frac{\Gamma(\gamma+k)}{\Gamma(\gamma)}
$$

In 2007,Shukla and Prajapati [11] introduced the function which is defined as,

$$
E_{\alpha, \beta}^{\gamma, q}(z)=\sum_{k=0}^{\infty} \frac{(\gamma)_{q k} z^{k}}{k ! \Gamma(\alpha k+\beta)} .
$$

where $z, \alpha, \beta, \gamma \in C, \min \{\operatorname{Re}(\alpha), \operatorname{Re}(\beta), \operatorname{Re}(\gamma)\}>0$, and $q \in(0,1) \cup N$

In 2012,further generalization of Mittag - Leffler function was defined by Salim [12] and Chauhan [2] as,

$$
E_{\alpha, \beta}^{\gamma, \delta, q}(z)=\sum_{k=0}^{\infty} \frac{(\gamma)_{q k} z^{k}}{(\delta)_{q k} \Gamma(\alpha k+\beta)} .
$$

where $z, \alpha, \beta, \gamma \in C, \min \{\operatorname{Re}(\alpha), \operatorname{Re}(\beta), \operatorname{Re}(\gamma)\}>0$, and $q \in(0,1) \cup N$

$$
(\gamma)_{q k}=\frac{\Gamma(\gamma+q k)}{\Gamma(\gamma)} \text { and }(\delta)_{q k}=\frac{\Gamma(\delta+q k)}{\Gamma(\delta)}
$$

denote the generalized Pochhammer symbol [11],

Definition 2.3 (6). The generalization of Mittag - Leffler function denoted by $Q_{\alpha, \beta, \delta}^{\gamma, q, r}(x)$ and defined by

$$
\begin{aligned}
Q_{\alpha, \beta, \delta}^{\gamma, q, r}(x) & =Q_{\alpha, \beta, \delta}^{\gamma, q, r}\left(a_{1}, a_{2}, \ldots, a_{r}, b_{1}, b_{2}, \ldots, b_{r}, x\right) \\
= & \sum_{s=0}^{\infty} \frac{\Pi_{n=1}^{r} \beta\left(b_{n}, s\right)(\gamma)_{q s}}{\Pi_{n=1}^{r} \beta\left(a_{n}, s\right)(\delta)_{q s} \Gamma(\alpha s+\beta)} x^{s},
\end{aligned}
$$

where $x, \alpha, \beta, \gamma, \delta, a_{i}, b_{i} \in C$, $\min \{\operatorname{Re}(\alpha), \operatorname{Re}(\beta), \operatorname{Re}(\gamma)\}>0$, and $q \in(0,1) \cup N$,

$$
(\gamma)_{q k}=\frac{\Gamma(\gamma+q k)}{\Gamma(\gamma)} \text { and }(\delta)_{q k}=\frac{\Gamma(\delta+q k)}{\Gamma(\delta)}
$$


Definition 2.4. (Riemann - Liouville Fractional Integral)The Riemann Liouville Fractional Integral of order $\alpha>0$ of function $f(t):(0, \infty) \rightarrow R$ is defined as,

$$
\left(I^{\alpha} f\right)(z)=\frac{1}{\Gamma(\alpha)} \int_{0}^{z}(z-t)^{\alpha-1} f(t) d t
$$

and

$$
\left(I_{a}^{\alpha} f\right)(z)=\frac{1}{\Gamma(\alpha)} \int_{a}^{z}(z-t)^{\alpha-1} f(t) d t
$$

Definition 2.5. The k - Riemann - Liouville Fractional Integral of order $\alpha, I_{k}^{\alpha} f$ where $\mathrm{f}$ is any function and $\alpha>0$, is given by [7],

$$
\left(I_{k}^{\alpha}\right)(z)=\frac{1}{k \Gamma_{k}(\alpha)} \int_{0}^{z}(z-t)^{\frac{a}{k}-1} f(t) d t
$$

This is the special case of [8],

$$
\left(I_{k, a}^{\alpha}\right)(z)=\frac{1}{k \Gamma_{k}(\alpha)} \int_{a}^{z}(z-t)^{\frac{\alpha}{k}-1} f(t) d t
$$

Property. [Holambe T.L. and Mohammed Mazhar-ul-Haque [4]] Let $\alpha>$ $0, \beta>0$ and $f$ be any function, then

$$
\begin{gathered}
I_{k}^{\alpha}\left(I_{k}^{\beta} f\right)(Z)=I_{k}^{\beta}\left(I_{k}^{\alpha} f\right)(z) \\
I_{k}^{\alpha}\left(I_{k}^{\beta} f\right)(Z)=I_{k}^{\beta}\left(I_{k}^{\alpha} f\right)(z)=\left(I_{k}^{\alpha+\beta} f\right)(z)=\frac{1}{k \Gamma_{k}(\alpha+\beta)} \int_{0}^{z}(z-t)^{\frac{\alpha+\beta}{k}-1} f(t) d t
\end{gathered}
$$

Definition 2.6. (Laplace Transform) The Laplace transform of integrable function $\mathrm{f}(\mathrm{t})$ is defined by

$$
L(f)(u)=\int_{0}^{\infty} e^{-u t} f(t) d t
$$

Definition 2.7. (Fourier Transform) The infinite Fourier Transform f(t) is defined as,

$$
F(f)(u)=\int_{-\infty}^{\infty} e^{i u t} f(t) d t
$$

Definition 2.8. (Fractional Fourier Transform)Fractional Fourier Transform [10], for $0<\alpha \leq 1$

$$
F_{\alpha}(f)(u)=\int_{-\infty}^{\infty} e^{i u \frac{1}{a} t} f(t) d t
$$


Definition 2.9. (Mellin Transform) The Mellin transform of $\mathrm{f}(\mathrm{x})$ over the interval $(0, \infty)$ is defined as,

$$
M[f(x)]=\overline{f(p)}=\int_{0}^{\infty} x^{p-1} f(x) d x, \text { where } p>0
$$

Definition 2.10. (Euler beta function)The beta function usually defined by

$$
B[f(t) ; a, b]=\int_{0}^{1} t^{a-1}(1-t)^{b-1} f(t) d t
$$

Definition 2.11. (Two-sided Laplace Transform)If $\mathrm{f}(\mathrm{t})$ is a real or complex valued function of the real variable $t$ defined for all real numbers, then the two-sided Laplace transform is defined by the integral

$$
\mathrm{B}(f)(u)=\int_{-\infty}^{\infty} e^{-u t} f(t) d t
$$

Definition 2.12. (Fourier Sine and Cosine Transform ) If $\mathrm{f}(\mathrm{t})$ is a real or complex valued function of the real variable $t$ defined for all real numbers, then the Fourier sine transform is defined by the integral

$$
F_{s}(f)(u)=\int_{-\infty}^{\infty} \sin (2 \pi u t) f(t) d t
$$

and the Fourier Cosine transform is defined by the integral

$$
F_{c}(f)(u)=\int_{-\infty}^{\infty} \cos (2 \pi u t) f(t) d t
$$

\section{Main Results}

Theorem 3.1. ( Laplace Transform) For any $x, \alpha, \beta, \gamma, \delta, a_{i}, b_{i} \in C$, $\min \{\operatorname{Re}(\alpha), \operatorname{Re}(\beta), \operatorname{Re}(\gamma), \operatorname{Re}(\delta)\}>0$, and $q \in(0,1) \cup N$, then the Laplace transform of generalized Mittag-Leffler function is

$$
L\left[Q_{\alpha, \beta, \delta}^{\gamma, q, r}(x)\right]=\frac{s !}{u} Q_{\alpha, \beta, \delta}^{\gamma, q, r}\left(u^{-1}\right)
$$

Proof. From the definition 2.3 of [6]

$$
Q_{\alpha, \beta, \delta}^{\gamma, q, r}(x)=\sum_{s=0}^{\infty} \frac{\Pi_{n=1}^{r} \beta\left(b_{n}, s\right)(\gamma)_{q s}}{\Pi_{n=1}^{r} \beta\left(a_{n}, s\right)(\delta)_{q s} \Gamma(\alpha s+\beta)} x^{s},
$$

and by definition 2.6 of Laplace Transform, 


$$
\begin{aligned}
& L(f)(u)=\int_{0}^{\infty} e^{-u t} f(t) d t \\
& L\left[Q_{\alpha, \beta, \delta}^{\gamma, q, r}(t)\right]=\int_{0}^{\infty} e^{-u t} Q_{\alpha, \beta, \delta}^{\gamma, q, r}(t) d t \\
& L\left[Q_{\alpha, \beta, \delta}^{\gamma, q, r}(t)\right]=\int_{0}^{\infty} e^{-u t} \sum_{s=0}^{\infty} \frac{\Pi_{n=1}^{r} \beta\left(b_{n}, s\right)(\gamma)_{q s}}{\Pi_{n=1}^{r} \beta\left(a_{n}, s\right)(\delta)_{q s} \Gamma(\alpha s+\beta)} t^{s} d t \\
& L\left[Q_{\alpha, \beta, \delta}^{\gamma, q, r}(t)\right]=\sum_{s=0}^{\infty} \frac{\Pi_{n=1}^{r} \beta\left(b_{n}, s\right)(\gamma)_{q s}}{\Pi_{n=1}^{r} \beta\left(a_{n}, s\right)(\delta)_{q s} \Gamma(\alpha s+\beta)} \int_{0}^{\infty} e^{-u t} t^{s} d t \\
& \text { since } \int_{0}^{\infty} e^{-u t} t^{s} d t=\frac{\Gamma(s+1)}{u^{s+1}}=\frac{s !}{u^{s+1}} \\
& L\left[Q_{\alpha, \beta, \delta}^{\gamma, q, r}(t)\right]=\sum_{s=0}^{\infty} \frac{\Pi_{n=1}^{r} \beta\left(b_{n}, s\right)(\gamma)_{q s}}{\prod_{n=1}^{r} \beta\left(a_{n}, s\right)(\delta)_{q s} \Gamma(\alpha s+\beta)} \frac{s !}{u^{s+1}} \\
& L\left[Q_{\alpha, \beta, \delta}^{\gamma, q, r}(t)\right]=\sum_{s=0}^{\infty} \frac{\Pi_{n=1}^{r} \beta\left(b_{n}, s\right)(\gamma)_{q s}}{\Pi_{n=1}^{r} \beta\left(a_{n}, s\right)(\delta)_{q s} \Gamma(\alpha s+\beta)} s ! u^{-(s+1)} \\
& L\left[Q_{\alpha, \beta, \delta}^{\gamma, q, r}(t)\right]=\frac{s !}{u} \sum_{s=0}^{\infty} \frac{\Pi_{n=1}^{r} \beta\left(b_{n}, s\right)(\gamma)_{q s}}{\Pi_{n=1}^{r} \beta\left(a_{n}, s\right)(\delta)_{q s} \Gamma(\alpha s+\beta)} u^{-s} \\
& L\left[Q_{\alpha, \beta, \delta}^{\gamma, q, r}(t)\right]=\frac{s !}{u} Q_{\alpha, \beta, \delta}^{\gamma, q, r}\left(u^{-1}\right)
\end{aligned}
$$

Theorem 3.2. (Fractional Fourier Transform) For any $x, \alpha, \beta, \gamma, \delta, a_{i}, b_{i} \in$ $C, \min \{\operatorname{Re}(\alpha), \operatorname{Re}(\beta), \operatorname{Re}(\gamma), \operatorname{Re}(\delta)\}>0$, and $q \in(0,1) \cup N$, then the Fractional Fourier transform of generalized Mittag-Leffler function is

$$
F_{\alpha}\left[Q_{\alpha, \beta, \delta}^{\gamma, q, r}(x)\right]=\sum_{s=0}^{\infty} \frac{s ! \Pi_{n=1}^{r} \beta\left(b_{n}, s\right)(\gamma)_{q s}(-1)^{-s} i^{-s-1} u^{\frac{-(s+1)}{\alpha}}}{\Pi_{n=1}^{r} \beta\left(a_{n}, s\right)(\delta)_{q s} \Gamma(\alpha s+\beta)}
$$

Proof. From the definition 2.3 of [6]

$$
Q_{\alpha, \beta, \delta}^{\gamma, q, r}(x)=\sum_{s=0}^{\infty} \frac{\Pi_{n=1}^{r} \beta\left(b_{n}, s\right)(\gamma)_{q s}}{\Pi_{n=1}^{r} \beta\left(a_{n}, s\right)(\delta)_{q s} \Gamma(\alpha s+\beta)} x^{s}
$$

and by definition of Fractional Fourier Transform, for $0<\alpha \leq 1$

$$
F_{\alpha}(f)(u)=\int_{-\infty}^{\infty} e^{i u \frac{1}{a} t} f(t) d t
$$




$$
\begin{gathered}
F_{\alpha}\left[Q_{\alpha, \beta, \delta}^{\gamma, q, r}(t)\right]=\int_{-\infty}^{\infty} e^{i u \frac{1}{a} t} Q_{\alpha, \beta, \delta}^{\gamma, q, r}(t) d t \\
F_{\alpha}\left[Q_{\alpha, \beta, \delta}^{\gamma, q, r}(t)\right]=\int_{-\infty}^{\infty} e^{i u \frac{1}{a} t} \sum_{s=0}^{\infty} \frac{\Pi_{n=1}^{r} \beta\left(b_{n}, s\right)(\gamma)_{q s}}{\Pi_{n=1}^{r} \beta\left(a_{n}, s\right)(\delta)_{q s} \Gamma(\alpha s+\beta)} t^{s} d t \\
F_{\alpha}\left[Q_{\alpha, \beta, \delta}^{\gamma, q, r}(t)\right]=\sum_{s=0}^{\infty} \frac{\Pi_{n=1}^{r} \beta\left(b_{n}, s\right)(\gamma)_{q s}}{\Pi_{n=1}^{r} \beta\left(a_{n}, s\right)(\delta)_{q s} \Gamma(\alpha s+\beta)} \int_{-\infty}^{\infty} e^{i u \frac{1}{a} t} t^{s} d t
\end{gathered}
$$

by substituting $i u^{\frac{1}{a}} t=-z, i u^{\frac{1}{a}} d t=-d z$

$$
\begin{gathered}
F_{\alpha}\left[Q_{\alpha, \beta, \delta}^{\gamma, q, r}(t)\right]=\sum_{s=0}^{\infty} \frac{\Pi_{n=1}^{r} \beta\left(b_{n}, s\right)(\gamma)_{q s}(-1)^{-s} i^{-s-1} u^{\frac{-(s+1)}{\alpha}}}{\Pi_{n=1}^{r} \beta\left(a_{n}, s\right)(\delta)_{q s} \Gamma(\alpha s+\beta)} \int_{0}^{\infty} e^{-z} z^{s} d z \\
F_{\alpha}\left[Q_{\alpha, \beta, \delta}^{\gamma, q, r}(t)\right]=\sum_{s=0}^{\infty} \frac{\Pi_{n=1}^{r} \beta\left(b_{n}, s\right)(\gamma)_{q s}(-1)^{-s} i^{-s-1} u^{\frac{-(s+1)}{\alpha}}}{\Pi_{n=1}^{r} \beta\left(a_{n}, s\right)(\delta)_{q s} \Gamma(\alpha s+\beta)} \Gamma(s+1) \\
F_{\alpha}\left[Q_{\alpha, \beta, \delta}^{\gamma, q, r}(x)\right]=\sum_{s=0}^{\infty} \frac{s ! \Pi_{n=1}^{r} \beta\left(b_{n}, s\right)(\gamma)_{q s}(-1)^{-s} i^{-s-1} u^{\frac{-(s+1)}{\alpha}}}{\Pi_{n=1}^{r} \beta\left(a_{n}, s\right)(\delta)_{q s} \Gamma(\alpha s+\beta)}
\end{gathered}
$$

Theorem 3.3. (Euler Beta Transform) For any $x, \alpha, \beta, \gamma, \delta, a_{i}, b_{i} \in C$, $\min \{\operatorname{Re}(\alpha), \operatorname{Re}(\beta), \operatorname{Re}(\gamma), \operatorname{Re}(\delta)\}>0$, and $q \in(0,1) \cup N$, then the Euler Beta transform of generalized Mittag-Leffler function is

$$
B\left[Q_{\alpha, \beta, \delta}^{\gamma, q, r}(t), a, b\right]=\sum_{s=0}^{\infty} \frac{\Pi_{n=1}^{r} \beta\left(b_{n}, s\right)(\gamma)_{q s}}{\Pi_{r=1}^{r} \beta\left(a_{n}, s\right)(\delta)_{q s} \Gamma(\alpha s+\beta)} \beta(s+a, b)
$$

Proof. from the definition 2.3 of [6],

$$
Q_{\alpha, \beta, \delta}^{\gamma, q, r}(x)=\sum_{s=0}^{\infty} \frac{\Pi_{n=1}^{r} \beta\left(b_{n}, s\right)(\gamma)_{q s}}{\Pi_{n=1}^{r} \beta\left(a_{n}, s\right)(\delta)_{q s} \Gamma(\alpha s+\beta)} x^{s}
$$

and by definition of Euler Beta function 2.10,

$$
\begin{gathered}
B[f(t) ; a, b]=\int_{0}^{1} t^{a-1}(1-t)^{b-1} f(t) d t \\
B\left[Q_{\alpha, \beta, \delta}^{\gamma, q, r}(t), a, b\right]=\int_{0}^{1} t^{a-1}(1-t)^{b-1} Q_{\alpha, \beta, \delta}^{\gamma, q, r}(t) d t
\end{gathered}
$$




$$
\begin{gathered}
B\left[Q_{\alpha, \beta, \delta}^{\gamma, q, r}(t), a, b\right]=\int_{0}^{1} t^{a-1}(1-t)^{b-1} \sum_{s=0}^{\infty} \frac{\Pi_{n=1}^{r} \beta\left(b_{n}, s\right)(\gamma)_{q s}}{\Pi_{n=1}^{r} \beta\left(a_{n}, s\right)(\delta)_{q s} \Gamma(\alpha s+\beta)} t^{s} d t \\
B\left[Q_{\alpha, \beta, \delta}^{\gamma, q, r}(t), a, b\right]=\sum_{s=0}^{\infty} \frac{\Pi_{n=1}^{r} \beta\left(b_{n}, s\right)(\gamma)_{q s}}{\Pi_{n=1}^{r} \beta\left(a_{n}, s\right)(\delta)_{q s} \Gamma(\alpha s+\beta)} \int_{0}^{1} t^{s+a-1}(1-t)^{b-1} d t \\
B\left[Q_{\alpha, \beta, \delta}^{\gamma, q, r}(t), a, b\right]=\sum_{s=0}^{\infty} \frac{\Pi_{n=1}^{r} \beta\left(b_{n}, s\right)(\gamma)_{q s}}{\Pi_{n=1}^{r} \beta\left(a_{n}, s\right)(\delta)_{q s} \Gamma(\alpha s+\beta)} \beta(s+a, s)
\end{gathered}
$$

Theorem 3.4. (Mellin Transform)For any $x, \alpha, \beta, \gamma, \delta, a_{i}, b_{i} \in C$, $\min \{\operatorname{Re}(\alpha), \operatorname{Re}(\beta), \operatorname{Re}(\gamma), \operatorname{Re}(\delta)\}>0$, and $q \in(0,1) \cup N$, then the Mellin transform of generalized Mittag-Leffler function is

$$
M\left[Q_{\alpha, \beta, \delta}^{\gamma, q, r}(x)\right]=\sum_{s=0}^{\infty} \frac{\Pi_{n=1}^{r} \beta\left(b_{n}, s\right)(\gamma)_{q s}}{\Pi_{r=1}^{r} \beta\left(a_{n}, s\right)(\delta)_{q s} \Gamma(\alpha s+\beta)} \int_{-\infty}^{\infty} e^{(u-s) t} d t
$$

Proof. from the definition 2.3 of [6],

$$
Q_{\alpha, \beta, \delta}^{\gamma, q, r}(x)=\sum_{s=0}^{\infty} \frac{\Pi_{n=1}^{r} \beta\left(b_{n}, s\right)(\gamma)_{q s}}{\Pi_{n=1}^{r} \beta\left(a_{n}, s\right)(\delta)_{q s} \Gamma(\alpha s+\beta)} x^{s}
$$

and by definition of Mellin Transform 2.9,

$$
\begin{gathered}
M[f(u)]=\int_{0}^{\infty} t^{u-1} f(t) d t \\
M\left[Q_{\alpha, \beta, \delta}^{\gamma, q, r}(t)\right]=\int_{0}^{\infty} t^{u-1} Q_{\alpha, \beta, \delta}^{\gamma, q, r}(t) d t \\
M\left[Q_{\alpha, \beta, \delta}^{\gamma, q, r}(t)\right]=\int_{0}^{\infty} t^{u-1} \sum_{s=0}^{\infty} \frac{\Pi_{n=1}^{r} \beta\left(b_{n}, s\right)(\gamma)_{q s}}{\Pi_{n=1}^{r} \beta\left(a_{n}, s\right)(\delta)_{q s} \Gamma(\alpha s+\beta)} t^{s} d t
\end{gathered}
$$

since by the relation,

$$
\begin{aligned}
& M(f)(u)=F\left[f\left(e^{-t}\right](-i u)=F(f)(u)=\int_{-\infty}^{\infty} e^{u t} f\left(e^{-t} d t\right.\right. \\
& M\left[Q_{\alpha, \beta, \delta}^{\gamma, q, r}(t)\right]=\int_{0}^{\infty} e^{u t} \sum_{s=0}^{\infty} \frac{\Pi_{n=1}^{r} \beta\left(b_{n}, s\right)(\gamma)_{q s}}{\Pi_{n=1}^{r} \beta\left(a_{n}, s\right)(\delta)_{q s} \Gamma(\alpha s+\beta)} e^{-s t} d t \\
& M\left[Q_{\alpha, \beta, \delta}^{\gamma, q, r}(t)\right]=\sum_{s=0}^{\infty} \frac{\Pi_{n=1}^{r} \beta\left(b_{n}, s\right)(\gamma)_{q s}}{\Pi_{n=1}^{r} \beta\left(a_{n}, s\right)(\delta)_{q s} \Gamma(\alpha s+\beta)} \int_{-\infty}^{\infty} e^{(u-s) t} d t
\end{aligned}
$$


Theorem 3.5. (Two-sided Laplace Transform)For any $x, \alpha, \beta, \gamma, \delta, a_{i}, b_{i} \in$ $C, \min \{\operatorname{Re}(\alpha), \operatorname{Re}(\beta), \operatorname{Re}(\gamma), \operatorname{Re}(\delta)\}>0$, and $q \in(0,1) \cup N$, then the Two-sided Laplace transform of generalized Mittag-Leffler function is

$$
\mathrm{B}\left[Q_{\alpha, \beta, \delta}^{\gamma, q, r}(x)\right]=\frac{s !}{u}\left\{Q_{\alpha, \beta, \delta}^{\gamma, q, r}\left(u^{-1}\right)+Q_{\alpha, \beta, \delta}^{\gamma, q, r}\left(\frac{-1}{u}\right)\right\}
$$

Proof. from the definition 2.3 of [6],

$$
Q_{\alpha, \beta, \delta}^{\gamma, q, r}(x)=\sum_{s=0}^{\infty} \frac{\Pi_{n=1}^{r} \beta\left(b_{n}, s\right)(\gamma)_{q s}}{\Pi_{n=1}^{r} \beta\left(a_{n}, s\right)(\delta)_{q s} \Gamma(\alpha s+\beta)} x^{s},
$$

and by definition of two-sided Laplace transform 2.11,

$$
\mathrm{B}(f)(u)=\int_{-\infty}^{\infty} e^{-u t} f(t) d t
$$

from the relationship between two-sided Laplace transform and Laplace transform,

$$
\begin{aligned}
& \mathrm{B}(f)(u)=L(f(t)(u)+L(f(-t)(-u) \\
& \mathrm{B}\left[Q_{\alpha, \beta, \delta}^{\gamma, q, r}(t)\right]=L\left(Q_{\alpha, \beta, \delta}^{\gamma, q, r}(t)\right)(u)+L\left(Q_{\alpha, \beta, \delta}^{\gamma, q, r}(-t)\right)(-u) \\
& \mathrm{B}\left[Q_{\alpha, \beta, \delta}^{\gamma, q, r}(t)\right]=\int_{0}^{\infty} e^{-u t} \sum_{s=0}^{\infty} \frac{\Pi_{n=1}^{r} \beta\left(b_{n}, s\right)(\gamma)_{q s}}{\Pi_{n=1}^{r} \beta\left(a_{n}, s\right)(\delta)_{q s} \Gamma(\alpha s+\beta)} t^{s} d t \\
& +\int_{0}^{\infty} e^{u t} \sum_{s=0}^{\infty} \frac{\Pi_{n=1}^{r} \beta\left(b_{n}, s\right)(\gamma)_{q s}}{\Pi_{n=1}^{r} \beta\left(a_{n}, s\right)(\delta)_{q s} \Gamma(\alpha s+\beta)}(-t)^{s} d t \\
& \mathrm{~B}\left[Q_{\alpha, \beta, \delta}^{\gamma, q, r}(t)\right]=\sum_{s=0}^{\infty} \frac{\Pi_{n=1}^{r} \beta\left(b_{n}, s\right)(\gamma)_{q s}}{\Pi_{n=1}^{r} \beta\left(a_{n}, s\right)(\delta)_{q s} \Gamma(\alpha s+\beta)} \int_{0}^{\infty} e^{-u t} t^{s} d t \\
& +\sum_{s=0}^{\infty} \frac{\Pi_{n=1}^{r} \beta\left(b_{n}, s\right)(\gamma)_{q s}(-1)^{s}}{\Pi_{n=1}^{r} \beta\left(a_{n}, s\right)(\delta)_{q s} \Gamma(\alpha s+\beta)} \int_{0}^{\infty} e^{-u t}(t)^{s} d t \\
& \text { since } \int_{0}^{\infty} e^{-u t} t^{s} d t=\frac{\Gamma(s+1)}{u^{(s+1)}}=\frac{s !}{u^{(s+1)}} \\
& \begin{aligned}
\mathrm{B}\left[Q_{\alpha, \beta, \delta}^{\gamma, q, r}(t)\right] & =\sum_{s=0}^{\infty} \frac{\Pi_{n=1}^{r} \beta\left(b_{n}, s\right)(\gamma)_{q s}}{\Pi_{n=1}^{r} \beta\left(a_{n}, s\right)(\delta)_{q s} \Gamma(\alpha s+\beta)} \frac{s !}{u^{(s+1)}} \\
& +\sum_{s=0}^{\infty} \frac{\Pi_{n=1}^{r} \beta\left(b_{n}, s\right)(\gamma)_{q s}(-1)^{s}}{\Pi_{n=1}^{r} \beta\left(a_{n}, s\right)(\delta)_{q s} \Gamma(\alpha s+\beta)} \frac{s !}{u^{(s+1)}}
\end{aligned}
\end{aligned}
$$




$$
\begin{aligned}
\mathrm{B}\left[Q_{\alpha, \beta, \delta}^{\gamma, q, r}(t)\right] & =\sum_{s=0}^{\infty} \frac{\Pi_{n=1}^{r} \beta\left(b_{n}, s\right)(\gamma)_{q s}}{\Pi_{n=1}^{r} \beta\left(a_{n}, s\right)(\delta)_{q s} \Gamma(\alpha s+\beta)} s ! u^{-(s+1)} \\
& +\sum_{s=0}^{\infty} \frac{\Pi_{n=1}^{r} \beta\left(b_{n}, s\right)(\gamma)_{q s}(-1)^{s}}{\Pi_{n=1}^{r} \beta\left(a_{n}, s\right)(\delta)_{q s} \Gamma(\alpha s+\beta)} s ! u^{-(s+1)} \\
\mathrm{B}\left[Q_{\alpha, \beta, \delta}^{\gamma, q, r}(t)\right] & =\frac{s !}{u} \sum_{s=0}^{\infty} \frac{\Pi_{n=1}^{r} \beta\left(b_{n}, s\right)(\gamma)_{q s}}{\Pi_{n=1}^{r} \beta\left(a_{n}, s\right)(\delta)_{q s} \Gamma(\alpha s+\beta)} u^{(-s)} \\
& +\frac{s !}{u} \sum_{s=0}^{\infty} \frac{\Pi_{n=1}^{r} \beta\left(b_{n}, s\right)(\gamma)_{q s}}{\Pi_{n=1}^{r} \beta\left(a_{n}, s\right)(\delta)_{q s} \Gamma(\alpha s+\beta)}\left(\frac{-1}{u}\right)^{s} \\
\mathrm{~B}\left[Q_{\alpha, \beta, \delta}^{\gamma, q, r}(x)\right] & =\frac{s !}{u}\left\{Q_{\alpha, \beta, \delta}^{\gamma, q, r}\left(u^{-1}\right)+Q_{\alpha, \beta, \delta}^{\gamma, q, r}\left(\frac{-1}{u}\right)\right\}
\end{aligned}
$$

Theorem 3.6. (Fourier Transform)For any $x, \alpha, \beta, \gamma, \delta, a_{i}, b_{i} \in C$, $\min \{\operatorname{Re}(\alpha), \operatorname{Re}(\beta), \operatorname{Re}(\gamma), \operatorname{Re}(\delta)\}>0$, and $q \in(0,1) \cup N$, then the Fourier transform of generalized Mittag-Leffler function is

$$
F\left[Q_{\alpha, \beta, \delta}^{\gamma, q, r}(x)\right]=\sum_{S=0}^{\infty} \frac{s ! \Pi_{n=1}^{r} \beta\left(b_{n}, s\right)(\gamma)_{q s}(-1)^{-s} i^{-s-1} u^{-(s+1)}}{\Pi_{n=1}^{r} \beta\left(a_{n}, s\right)(\delta)_{q s} \Gamma(\alpha s+\beta)}
$$

Proof. from the definition 2.3 of [6],

$$
Q_{\alpha, \beta, \delta}^{\gamma, q, r}(x)==\sum_{s=0}^{\infty} \frac{\Pi_{n=1}^{r} \beta\left(b_{n}, s\right)(\gamma)_{q s}}{\Pi_{n=1}^{r} \beta\left(a_{n}, s\right)(\delta)_{q s} \Gamma(\alpha s+\beta)} x^{s}
$$

and by definition of Fourier Transform 2.7,

$$
\begin{gathered}
F(f)(u)=\int_{-\infty}^{\infty} e^{i u t} f(t) d t \\
F\left[Q_{\alpha, \beta, \delta}^{\gamma, q, r}(t)\right]=\int_{-\infty}^{\infty} e^{i u t} Q_{\alpha, \beta, \delta}^{\gamma, q, r}(t) d t \\
F\left[Q_{\alpha, \beta, \delta}^{\gamma, q, r}(t)\right]=\int_{-\infty}^{\infty} e^{i u t} \sum_{s=0}^{\infty} \frac{\Pi_{n=1}^{r} \beta\left(b_{n}, s\right)(\gamma)_{q s}}{\Pi_{n=1}^{r} \beta\left(a_{n}, s\right)(\delta)_{q s} \Gamma(\alpha s+\beta)} t^{s} d t \\
F\left[Q_{\alpha, \beta, \delta}^{\gamma, q, r}(t)\right]=\sum_{s=0}^{\infty} \frac{\Pi_{n=1}^{r} \beta\left(b_{n}, s\right)(\gamma)_{q s}}{\Pi_{n=1}^{r} \beta\left(a_{n}, s\right)(\delta)_{q s} \Gamma(\alpha s+\beta)} \int_{-\infty}^{\infty} e^{i u t} t^{s} d t
\end{gathered}
$$


by substituting $i u t=-z, i u d t=-d z$,

$$
\begin{gathered}
F\left[Q_{\alpha, \beta, \delta}^{\gamma, q, r}(t)\right]=\sum_{s=0}^{\infty} \frac{\Pi_{n=1}^{r} \beta\left(b_{n}, s\right)(\gamma)_{q s}(-1)^{-s} i^{-s-1} u^{-(s+1}}{\Pi_{n=1}^{r} \beta\left(a_{n}, s\right)(\delta)_{q s} \Gamma(\alpha s+\beta)} \int_{0}^{\infty} e^{-z} z^{s} d z \\
F\left[Q_{\alpha, \beta, \delta}^{\gamma, q, r}(t)\right]=\sum_{s=0}^{\infty} \frac{\Pi_{n=1}^{r} \beta\left(b_{n}, s\right)(\gamma)_{q s}(-1)^{-s} i^{-s-1} u^{-(s+1}}{\Pi_{n=1}^{r} \beta\left(a_{n}, s\right)(\delta)_{q s} \Gamma(\alpha s+\beta)} \Gamma(s+1) \\
F\left[Q_{\alpha, \beta, \delta}^{\gamma, q, r}(t)\right]=\sum_{s=0}^{\infty} \frac{s ! \Pi_{n=1}^{r} \beta\left(b_{n}, s\right)(\gamma)_{q s}(-1)^{-s} i^{-s-1} u^{-(s+1)}}{\Pi_{n=1}^{r} \beta\left(a_{n}, s\right)(\delta)_{q s} \Gamma(\alpha s+\beta)}
\end{gathered}
$$

Theorem 3.7. ( Fourier Sine and Cosine Transform) For any $x, \alpha, \beta, \gamma, \delta$, $a_{i}, b_{i} \in C, \min \{\operatorname{Re}(\alpha), \operatorname{Re}(\beta), \operatorname{Re}(\gamma), \operatorname{Re}(\delta)\}>0$, and $q \in(0,1) \cup N$, then the Fourier sine and cosine transform of generalized Mittag-Leffler function is

$$
F_{c}\left[Q_{\alpha, \beta, \delta}^{\gamma, q, r}(t)\right]=\operatorname{Realpart}\left\{\sum_{S=0}^{\infty} \frac{s ! \Pi_{n=1}^{r} \beta\left(b_{n}, s\right)(\gamma)_{q s}(-1)^{-s}(i 2 \pi)^{-s-1} u^{-(s+1)}}{\Pi_{n=1}^{r} \beta\left(a_{n}, s\right)(\delta)_{q s} \Gamma(\alpha s+\beta)}\right\}
$$

and

$F_{s}\left[Q_{\alpha, \beta, \delta}^{\gamma, q, r}(t)\right]=\operatorname{Imaginarypart}\left\{\sum_{S=0}^{\infty} \frac{s ! \Pi_{n=1}^{r} \beta\left(b_{n}, s\right)(\gamma)_{q s}(-1)^{-s}(i 2 \pi)^{-s-1} u^{-(s+1)}}{\Pi_{n=1}^{r} \beta\left(a_{n}, s\right)(\delta)_{q s} \Gamma(\alpha s+\beta)}\right\}$

Proof. from the definition 2.3 of [6],

$$
Q_{\alpha, \beta, \delta}^{\gamma, q, r}(x)=\sum_{s=0}^{\infty} \frac{\Pi_{n=1}^{r} \beta\left(b_{n}, s\right)(\gamma)_{q s}}{\Pi_{n=1}^{r} \beta\left(a_{n}, s\right)(\delta)_{q s} \Gamma(\alpha s+\beta)} x^{s}
$$

and by definition of Fourier sine and cosine transform 2.12,

$$
\begin{gathered}
F_{s}(f)(u)=\int_{-\infty}^{\infty} \sin (2 \pi u t) f(t) d t \\
F_{s}\left[Q_{\alpha, \beta, \delta}^{\gamma, q, r}(t)\right]=\int_{-\infty}^{\infty} \sin (2 \pi u t) Q_{\alpha, \beta, \delta}^{\gamma, q, r}(t) d t \\
F_{s}\left[Q_{\alpha, \beta, \delta}^{\gamma, q, r}(t)\right]=\int_{-\infty}^{\infty} \sin (2 \pi u t) \sum_{s=0}^{\infty} \frac{\Pi_{n=1}^{r} \beta\left(b_{n}, s\right)(\gamma)_{q s}}{\prod_{n=1}^{r} \beta\left(a_{n}, s\right)(\delta)_{q s} \Gamma(\alpha s+\beta)} t^{s} d t
\end{gathered}
$$




$$
F_{s}\left[Q_{\alpha, \beta, \delta}^{\gamma, q, r}(t)\right]=\sum_{s=0}^{\infty} \frac{\Pi_{n=1}^{r} \beta\left(b_{n}, s\right)(\gamma)_{q s}}{\Pi_{n=1}^{r} \beta\left(a_{n}, s\right)(\delta)_{q s} \Gamma(\alpha s+\beta)} \int_{-\infty}^{0} \sin (2 \pi u t) t^{s} d t
$$

and

$$
\begin{gathered}
F_{c}(f)(u)=\int_{-\infty}^{\infty} \cos (2 \pi u t) f(t) d t \\
F_{c}\left[Q_{\alpha, \beta, \delta}^{\gamma, q, r}(t)\right]=\int_{-\infty}^{\infty} \cos (2 \pi u t) Q_{\alpha, \beta, \delta}^{\gamma, q, r}(t) d t \\
F_{c}\left[Q_{\alpha, \beta, \delta}^{\gamma, q, r}(t)\right]=\int_{-\infty}^{\infty} \cos (2 \pi u t) \sum_{s=0}^{\infty} \frac{\Pi_{n=1}^{r} \beta\left(b_{n}, s\right)(\gamma)_{q s}}{\Pi_{n=1}^{r} \beta\left(a_{n}, s\right)(\delta)_{q s} \Gamma(\alpha s+\beta)} t^{s} d t \\
F_{c}\left[Q_{\alpha, \beta, \delta}^{\gamma, q, r}(t)\right]=\sum_{s=0}^{\infty} \frac{\Pi_{n=1}^{r} \beta\left(b_{n}, s\right)(\gamma)_{q s}}{\Pi_{n=1}^{r} \beta\left(a_{n}, s\right)(\delta)_{q s} \Gamma(\alpha s+\beta)} \int_{-\infty}^{0} \cos (2 \pi u t) t^{s} d t
\end{gathered}
$$

since $e^{-i 2 \pi u t}=\cos (2 \pi u t)-i \sin (2 \pi u t)$

$$
F\left[Q_{\alpha, \beta, \delta}^{\gamma, q, r}(t)\right]=\sum_{s=0}^{\infty} \frac{\Pi_{n=1}^{r} \beta\left(b_{n}, s\right)(\gamma)_{q s}}{\Pi_{n=1}^{r} \beta\left(a_{n}, s\right)(\delta)_{q s} \Gamma(\alpha s+\beta)} \int_{-\infty}^{0} e^{-i 2 \pi u t} t^{s} d t
$$

by substituting $i 2 \pi u t=z, i 2 \pi u d t=d z$

$$
\begin{gathered}
F\left[Q_{\alpha, \beta, \delta}^{\gamma, q, r}(t)\right]=\sum_{S=0}^{\infty} \frac{\Pi_{n=1}^{r} \beta\left(b_{n}, s\right)(\gamma)_{q s}(-1)^{-s}(i 2 \pi)^{-s-1} u^{-(s+1)}}{\Pi_{n=1}^{r} \beta\left(a_{n}, s\right)(\delta)_{q s} \Gamma(\alpha s+\beta)} \int_{0}^{\infty} e^{-z} z^{s} d z \\
F\left[Q_{\alpha, \beta, \delta}^{\gamma, q, r}(t)\right]=\sum_{S=0}^{\infty} \frac{\Pi_{n=1}^{r} \beta\left(b_{n}, s\right)(\gamma)_{q s}(-1)^{-s}(i 2 \pi)^{-s-1} u^{-(s+1)}}{\Pi_{n=1}^{r} \beta\left(a_{n}, s\right)(\delta)_{q s} \Gamma(\alpha s+\beta)} \Gamma(s+1) \\
F\left[Q_{\alpha, \beta, \delta}^{\gamma, q, r}(t)\right]=\sum_{S=0}^{\infty} \frac{s ! \Pi_{n=1}^{r} \beta\left(b_{n}, s\right)(\gamma)_{q s}(-1)^{-s}(i 2 \pi)^{-s-1} u^{-(s+1)}}{\Pi_{n=1}^{r} \beta\left(a_{n}, s\right)(\delta)_{q s} \Gamma(\alpha s+\beta)}
\end{gathered}
$$

thus

$$
F_{c}\left[Q_{\alpha, \beta, \delta}^{\gamma, q, r}(t)\right]=\operatorname{Realpart}\left\{\sum_{S=0}^{\infty} \frac{s ! \Pi_{n=1}^{r} \beta\left(b_{n}, s\right)(\gamma)_{q s}(-1)^{-s}(i 2 \pi)^{-s-1} u^{-(s+1)}}{\Pi_{n=1}^{r} \beta\left(a_{n}, s\right)(\delta)_{q s} \Gamma(\alpha s+\beta)}\right\}
$$

and

$$
F_{s}\left[Q_{\alpha, \beta, \delta}^{\gamma, q, r}(t)\right]=\operatorname{Imaginarypart}\left\{\sum_{S=0}^{\infty} \frac{s ! \Pi_{n=1}^{r} \beta\left(b_{n}, s\right)(\gamma)_{q s}(-1)^{-s}(i 2 \pi)^{-s-1} u^{-(s+1)}}{\Pi_{n=1}^{r} \beta\left(a_{n}, s\right)(\delta)_{q s} \Gamma(\alpha s+\beta)}\right\}
$$




\section{Conclusion}

The Laplace transform, Fourier transform, Fourier sine and cosine transform, Euler beta and the Mellin transform for generalized Mittag-Leffler function were derived.

\section{References}

[1] Agarwal R.P., A propos d'une note M. Pierre Humbert,C.R.Acad.Sci. Paris 236 (1953),pp.2031-2032.

[2] Chouhan Amit and Satish saraswat, Some Remarks on Generalized Mittag-Leffler Function and Fractional operators,IJMMAC Vol2, No.2, pp. 131-139.

[3] GM.Mittag - Leffler, Sur la nouvelle function of $E_{\alpha}(x)$, C.R.Acad. Sci. Paris 137 (1903), pp.554-558.

[4] Holambe T.L.and Mohammed Mazhar -ul- Haque, A remark on semigroup property in fractional calculus, International Journal of Mathematics and computer Application Research, 4(6),2014,pp.27-32.

[5] Humbert P. and Agarwal R.P., Sur la function de Mittag - Leffler et quelquesunes deses generalizations, Bull. Sci.Math. (2)77(1953), pp.180-186.

[6] Mohammed Mazhar-ul- Haque and Holambe T.L., A Q function in fractional calculus, Journal of Basic and Applied Research International, International knowledge press, vol. 6, issue 4 (2015),pp.248-252.

[7] Mubeen S.and Habibullah G.M., k-Fractional Integrals and Applications, Int. J. Compt. Math. Science, 7(2)(2012),pp.89-94.

[8] Podlubny I., Fractional Differential Equations, Academic Press, San Diego, CaliforniaU.S.A., (1999).

[9] Prabhakar T.R., A Singular Integral Equation with a generalized Mittag - Leffler function in the kernel, Yokohama Math. J. 19(1971), PP.7-15.

[10] Romero L., Cerutti R.and Luque L., A New Fractional Fourier Transform and convolutions products, International Journal of Pure and Applied Mathematics,66(2011),pp.397408.

[11] Shukla A.K. and Prajapati J.C., On a generalization of Mittag - Leffler function and its properties, J.Math.Anal.Appl.336(2007),pp.79-81.

[12] Salim T.O. and Faraj O., A generalization of Mittag-Leffler function and Integral operator associated with the Fractional calculus, Journal of Fractional Calculus and Applications,3(5) (2012),pp.1-13.

[13] Wiman A., Uber de fundamental satz in der theorie der funktionen, Acta Math. 29(1905),pp.191-201. 
